\title{
INTEGRATED REHABILITATION IN CHRONIC PAIN
}

\author{
Younes Alì ${ }^{1}$, Barassi Giovanni
}

${ }^{1}$ MD, Anesthesiology and Algology Specialist, Sector of Clinical Evaluation and Diagnosis of Ce.Fi.R.R. venue "G. d'Annunzio" University of Chieti Pescara (Chieti-IT); Clinical Manager International Association for Fibromyalgia Research.

${ }^{2}$ PhD, Coordinator of the Physiotherapy Center for Rehabilitation and Reeducation (Ce.Fi.R.R.) venue "G. d'Annunzio" University of Chieti-Pescara (Chieti-IT); ITC Project Coordinator (Integrated Thermal Cares) Castelnuovo della Daunia Medical Thermal Center (Foggia-IT).

KEYWORDS: Chronic Pain, Manual Therapy, Eclectrotherapy, Magnetic Field Therapy, Physiotherapy

\section{ABSTRACT}

A rehabilitation protocol has been developed at the Rehabilitation and Rehabilitation Physiotherapy Center, University of Chieti-Pescara, which provides for the combined use of Frems, Manual Therapy and Cyclotronic Bioresonance on patients with chronic pain.

Chronic pain management represents one of the most important health challenges of the 21 st century. According to surveys, it is estimated that one in five Europeans suffers from it and that $26 \%$ of the Italian population is affected by this pathological status, to the point of believing its treatment has become a priority for our national health system. Pain - which is nothing but one of the symptomatic manifestations of the disease, therefore a consequence of a preexisting / previous pathological state - represents one of the main discomforts as regards the life of patients, being, in this perspective, the due to a significant deterioration in the quality of the same. Improper or absent pain management creates significant physical, psychological and social effects; moreover, considering the lost working days, it can also have an important economic impact.

The identification of a therapeutic model capable of guaranteeing effective analgesic treatment for the population is certainly a crucial objective, which can only be achieved through a better preparation of the medical profession, as well as promoting the creation of appropriate service networks, aimed at involve all health professionals.

\section{INTRODUCTION}

Pain is a global medical problem that can cause longterm disability. Pain perception is supported by the transduction of mechanical, thermal and chemical sensory inputs into the subjective awareness of pain (Tsang et al., 2008).

In particular, the impact of an individual's chronic pain has repercussions on the family sphere, such as in marital relations and / or in economic terms, where a comfortable working situation must be renounced; even worse when one of the family members themselves must take on the role of caregiver for the person affected by the problem and associated disabilities (Bevan et al., 2009).

In addition, numerous studies have highlighted the importance of the behavior of family members towards the person suffering from chronic pain, in particular of the partner, which can be vital for positive or negative results, for example prompt responses (being excessively useful with tasks and duties), mood influences and negative reactions (anger and frustration in partners) that affects the quality of the relationship (Leonard, 2006; Hooper \& Ong, 2005).

There is also evidence of more direct biological and genetic influences and interactions between family members. Numerous studies on twins and family have reported concordance of shared biological inheritance (shared risk) between family members for pain conditions (Hartvigsen et al., 2009).

For example, Hocking et al. report that the genetic inheritance estimate for chronic pain was $29 \%$ in a study of 2,195 extended families and another study, which showed a significant association between chronic maternal and adolescent chronic pain (Hocking et al., 2012).

The pain pathways include a complex sensory system, which is activated to provide protective responses to harmful stimuli. Input related to harmful stimuli is transmitted by nociceptors from primary afferent fibers $\mathrm{A} \delta$ and $\mathrm{C}$. These fibers have cell bodies located in the dorsal root ganglion and synapses with neurons in the spinal dorsal horn. Various neurotransmitters such as glutamate, the calcitonin gene-related peptide (CGRP) and substance $P$ are released as part of the signal transduction. Brain structures, including the primary somatosensory cortex, secondary somatosensory cortex, anterior cingulate cortex (ACC), prefrontal cortex (PFC), insular cortex, amygdala, thalamus, cerebellum and PAG have been identified as regions associated with pain perception (Reddi et al., 2013).

These brain regions are not activated separately; they are functionally linked and contribute in a combined way to the processing of pain. Changes in emotional and motivational cues can affect the intensity and degree of pain experience.

Neurotransmitters are chemicals that mediate the transmission of impulses through the synapse. The transmission of neuronal signals through the synapse began with the release of neurotransmitters from the presynaptic neuron. Neurotransmitters are released into the synaptic cleft and bind to neurotransmitter receptors on postsynaptic neurons. Neurotransmitters can be classified based on their function (excitatory or inhibitory), their molecular size (small molecules, including amino acids and monoamines, or large molecules, including peptides), or their type (inflammato- 
ry mediators, including prostaglandin E2, adenosine triphosphate, adenosine, histamine, glutamate and nitric oxide (NO) or non-inflammatory mediators, including GABA, CGRP, peptides, glycine and cannabinoids) (Yam et al., 2018).

The binding of neurotransmitters to their receptors on postsynaptic neurons influences the transmission of pain in an inhibitory or excitatory way (Ji \& Suter, 2007).

Glial cells, such as microglia and astrocytes, release several neurotransmitters that contribute to the development and maintenance of chronic pain by activating or deactivating the nociceptive neurons in the CNS.

Chronic pain is one of the most intractable clinical problems faced by doctors and can be physically and emotionally debilitating. Combinations of treatments are currently used for the treatment of chronic pain, but a subset of patients experience persistent tolerable pain. Therefore, a deeper understanding of the mechanisms and key factors involved in chronicizing pain is needed to identify new therapeutic targets for developing better treatments for chronic pain (Carniglia et al., 2017).

A group of 10 subjects suffering from chronic pain and fibromyalgia were examined at the physiotherapy, rehabilitation and re-education center at the University of Chieti.

They were treated through an integrated peripheral sensorineural stimulation path that included:

- FREMSTM, an electrotherapy which, due to its fundamental characteristics, is able to systematically influence functional subcutaneous structures, producing determined and repetitive events such as vasomotion, understood as the pulsatile rhythmic activity of the vessels, through the involvement of the precapillary sphincters of the microcirculation;

- Manual therapy with osteopathic modalities;

- Cyclotronic bioresonance: The Quec phisis system.

\section{METHODS AND MATERIALS}

10 patients suffering from chronic pain and fibromyalgia syndrome were selected at the Physiotherapy and Rehabilitation and Rehabilitation Center, University of Chieti-Pescara.

Studies using selected criteria for the diagnosis of fibromyalgia were selected.

Recently, the American College of Rheumatology (ACR) has introduced new criteria (Wolfe 2010 and Wolfe 2011); however, the American College of Rheumatology (ACR) 1990 (Wolfe 1990) criteria have been the dominant diagnostic criteria used for the diagnosis of fibromyalgia over the past two decades.

The 1990 ACR criteria include: a) diffuse pain lasting more than three months; b) digital palpation pain with pressure of $4 \mathrm{~kg}$ in at least 11 of the 18 sites of specified sensitive points.

Other published criteria are: Smythe 1981, Yunus 1981, Yunus 1982 and Yunus 1984. Although there are some differences between the diagnostic criteria, all of them were considered acceptable and comparable for the purpose of this study.

The inclusion criteria considered were:

- pain spread for six months or more and to be diagnosed with FM according to the ACR 1990 classification criteria;

- normal neurological examination results, including deep tendon reflexes, voluntary muscle action and sensory function;

- not obtaining improvements as a result of other treatments including nonsteroidal anti-inflammatory drugs, opioids, tricyclic antidepressants (amitriptyline or cyclobenzaprine), selective serotonin reuptake inhibitors, serotonin-norepinephrine reuptake inhibitors, anticonvulsant drugs such as gabapentine, pregalin some other multidisciplinary therapies.

The exclusion criteria were:

- pacemaker;

- $\quad$ autoimmune or inflammatory diseases;

- participation in other clinical trials;

- pregnancy or breastfeeding;

- diabetes mellitus, neurological pathologies, alcoholism, polyneuropathy, renal failure, asthma, emphysema, bronchitis, epilepsy, schizophrenia, or psychosis.

Following inclusion and exclusion criteria of the patients enrolled for the study, 10 women with an average age of 43 years.

All participants signed the informed consent for the experimental procedure, which complies with the latest revision of the Helsinki Declaration and with the procedures defined by the ISO 9001 standards for "Research and Experimentation"; this procedure also protects the privacy of subjects participating in biomedical research.

All patients underwent three times a week for three months to:

- Therapy of the frequency rhythmic electric modulation system (FREMS) (FT), based on the administration of pulsed, negative, asymmetric single-phase pulsed current electric fields, generated by a neurostimulator with the characteristics of variable low frequency, high voltage and duration of the very low impulse.

- Manual therapy with osteopathic modality; pragmatically, the study protocol used the listed techniques:

- Joint treatment, balanced ligament tension / joint ligament tension treatment, counter-stretching treatment, direct treatment, muscle energy treatment, soft tissue treatment (ST) and visceral manipulative treatment (Cerritelli et al., 2020). These techniques include most techniques used in patients with chronic pain.

- Cyclotronic bioresonance: the Quec phisis system emits ultra-weak electromagnetic waves which, by calculating the cyclotronic resonance of the ions in the domains of water coherence, excite the ions themselves making them "bioavailable". The emission of highly targeted and personalized magnetic signals can: Stimulate the biological functions of cells Rebalance the correct membrane potential Activates enzymatic processe Restores proper ion exchange for cellular metabolism Promote intra-extra cellular ionic balance (Islamov et al., 2002). 


\begin{tabular}{|c|c|c|c|c|c|}
\hline \multicolumn{3}{|l|}{ FIQR } & \multicolumn{3}{|l|}{ VAS } \\
\hline & pre & post & & pre & post \\
\hline pz 1 & 60,1 & 49,7 & pz 1 & 7,0 & 5 \\
\hline pz 2 & 75,4 & 30,1 & pz 2 & 8 & 3 \\
\hline pz 3 & 60,2 & 49,3 & pz 3 & 8,3 & 5 \\
\hline pz 4 & 79,4 & 55,2 & pz 4 & 9,7 & 4,6 \\
\hline pz 5 & 88,5 & 52,3 & pz 5 & 8 & 5,5 \\
\hline pz 6 & 65,2 & 49,4 & pz 6 & 8,7 & 6,2 \\
\hline pz 7 & 79,4 & 53,1 & pz 7 & 9 & 5,8 \\
\hline pz 8 & 76,3 & 65,4 & pz 8 & 7,7 & 4,5 \\
\hline pz 9 & 82,3 & 45,2 & pz 9 & 8,8 & 4,9 \\
\hline pz 10 & 72,5 & 55,2 & pz 10 & 9 & 6,8 \\
\hline Mean & 73,9 & 50,5 & Mean & 8,4 & 5,1 \\
\hline$\% \mathrm{mgl}$ & 31,7 & & $\% \mathrm{mgl}$ & 39 & \\
\hline T.Test & 0,000 & & T.Test & 0,00 & \\
\hline
\end{tabular}

Tab. 1

\begin{tabular}{|c|c|c|c|c|c|c|c|c|}
\hline & \multicolumn{4}{|c|}{ Trapezius } & \multicolumn{4}{|c|}{ Lumbar paravertebrals (L3) } \\
\hline & \multicolumn{2}{|l|}{$\mathrm{dx}$} & \multicolumn{2}{|l|}{ sx } & \multicolumn{2}{|l|}{$\mathrm{dx}$} & \multicolumn{2}{|l|}{ sx } \\
\hline & pre & post & pre & post & pre & post & pre & post \\
\hline pz 1 & 16,4 & 12,3 & 17,5 & 12 & 12,8 & 11 & 12,7 & 11,9 \\
\hline pz 2 & 14,5 & 13,4 & 12 & 13,2 & 14,5 & 12 & 15 & 12,9 \\
\hline pz 3 & 14,5 & 12,3 & 13,4 & 12,5 & 13,4 & 13 & 13,9 & 12 \\
\hline pz 4 & 17,5 & 18,2 & 12,1 & 11,5 & 15,3 & 13,2 & 14,2 & 11,3 \\
\hline pz 5 & 13,9 & 11,8 & 13 & 12,3 & 15,6 & 12,1 & 13,8 & 12 \\
\hline pz 6 & 13 & 12,5 & 12,1 & 12,5 & 16,3 & 13,8 & 14,4 & 12,3 \\
\hline pz 7 & 14,1 & 11,5 & 13,3 & 11,4 & 14,5 & 12,2 & 13,5 & 11,2 \\
\hline pz 8 & 16,8 & 15,9 & 19,1 & 15,5 & 16,2 & 13 & 18,5 & 15,3 \\
\hline pz 9 & 13,2 & 12,5 & 15,3 & 12,3 & 15,2 & 13,3 & 15,1 & 11,9 \\
\hline pz 10 & 11 & 9,8 & 13,5 & 10 & 16,2 & 12,5 & 14,2 & 10,3 \\
\hline Mean & 14,6 & 13,0 & 14,1 & 12,4 & 15 & 12,8 & 14,5 & 12,1 \\
\hline$\% \mathrm{mgl}$ & \multicolumn{2}{|l|}{11,0} & \multicolumn{2}{|l|}{12,6} & \multicolumn{2}{|l|}{14,7} & \multicolumn{2}{|l|}{16,5} \\
\hline T.Test & \multicolumn{2}{|c|}{0,0089} & \multicolumn{2}{|c|}{0,094} & \multicolumn{2}{|c|}{0,000386} & \multicolumn{2}{|l|}{0,001} \\
\hline
\end{tabular}

Tab. 2

\section{RESULTS}

The Analysis of results has been performed through the Office Excel software (Microsoft $\AA$, Redmond, Washington, USA). For each parameter taken into consideration, the mean, standard deviation, percentage improvement and Student's T-Test were calculated. In particular, for the rating scale, the improvement percentages were significant (FIQR: $31.7 \%$, VAS: $39 \%$, $\mathrm{P}$ value $<0,05)$, indicating an improvement of both symptoms of fibromyalgia and perceived pain (Table 1).
The rheological values related to myometry positively changed for muscle tone which showed a significant reduction and rebalancing (Table 2 ).

Stiffness is the measure of resistance when force is exerted on an elastic object or body (Table 3). Stiffness is not measured by the properties of the rubber ball, but by a force independent of the object.

Considering that elasticity is different from stiffness because it measures the ability of an object to resist deformation and that elasticity is a property of a material that represents its ability to completely regain

\begin{tabular}{|l|l|l|l|l|l|l|l|l|}
\hline \multicolumn{2}{|l|}{ Table 3-Stiffness variations } \\
\hline
\end{tabular}

Tab.3 


\begin{tabular}{|l|l|l|l|l|l|l|l|l|l|}
\hline \multicolumn{7}{|l|}{ Table 4- Decrement variations } \\
\hline
\end{tabular}

Tab.4

the original shape and size after removing the load applied, the results obtained are to be considered positive (Table 4).

The change in values was statistically significant across the study group.

\section{DISCUSSION}

The size of chronic pain still represents a challenge for doctors and rehabilitators and one of the main health problems worldwide, both for the aging of the population and for the increase in chronic-degenerative diseases. The size of chronic pain, its socio-economic impact and the obvious gaps in the care front are priority areas of intervention for European and national health policies. Properly treating pain allows not only to significantly improve the quality of life, but also to prevent secondary disabilities (Alford et al., 2008). A correct approach to pain treatment should involve health professionals by creating an efficient network between hospital, clinic or medical center and the local area. Consequently, it becomes a necessity not only to pay attention to the clinical health part, but also to promote cultural change, in order to deal with relational and emotional problems with the right awareness, which can interfere with the success of the therapeutic program itself. .

First of all, institutions are called to respond and take action to create socio-economic political assumptions and provide useful tools for the success of an integrated and multidisciplinary approach, which strengthens a network of connections between specialists in the sector, where the patient and his recovery / cure is placed at the center of the patient empowerment.

\section{CONCLUSION}

This integrated approach has proven to be a valid and effective path with respect to the parameters examined, in particular as regards the therapeutic integration of the various treatment methods represented by: cyclonic resonance resonance at low electromagnetic frequencies (QUEC PHISIS), manual therapy and FREMS.

\section{REFERENCES}

1. Alford, D. P., Liebschutz, J., Chen, I. A., et al. (2008). Update in Pain Medicine. Gen Intern Med., 23(6):841-845.

2. Bevan, S., Quadrello, T., McGee, R., Mahdon, M., Vavrovsky, A., \& Barham, L. (2009). Fit for work. Musculoskeletal disorders in the European workforce. London: The work foundation, 2009.

3. Carniglia, L., Ramirez, D., Durand, D., Saba, J., Turati, J., Caruso, C., et al. (2017). Neuropeptides and Microglial Activation in Inflammation, Pain, and Neurodegenerative Diseases. Mediat. Inflamm., 2017:5048616.

4. Cerritelli, F., Chiacchiaretta, P., Gambi, F., Perrucci, M. G., Barassi, G., Visciano, C., Bellomo, R. G., Saggini, R. \& Ferretti, A.(2020). Effect of manual approaches with osteopathic modality on brain correlates of interoception: an fMRI study. Sci Rep;10(1):3214.

5. Hooper, H. \& Ong, B. N. (2005). When Harry met Barry, and other stories: A partner's influence on relationships in back pain care. Anthropol Med., 12(1):47-60. 
6. Hartvigsen, J., Nielsen, J., Kyvik, K. O., Fejer, R., Vach, W., Iachine, I. \& Leboeuf, Y. de C.(2009). Heritability of spinal pain and consequences of spinal pain: a comprehensive genetic epidemiologic analysis using a population-based sample of 15,328 twins ages 20-71 years. Arthritis Care Res, 61:1343-51.

7. Hocking, L. J., Morris, A. D., Dominiczak, A. F., Porteous, D. J., \& Smith, B. H. (2012). Heritability of chronic pain in 2195 extended families. Eur J Pain, 16:1053-63.

8. Islamov, B. I., Balabanova, R. M., Funtikov, V. A., Gotovskii, Y. V. \& Meizerov, E. (2002). Effect of bioresonance therapy on antioxidant system in lymphocytes in patients with rheumatoid arthritis. Bull Exp Biol Med., 134(3):24850.

9. Ji, R. R., \& Suter, M. R. (2007). p38 MAPK, microglial signaling, and neuropathic pain. Mol. Pain., 3:33.

10. Leonard, M. T., Cano, A. \& Johansen, A. B. (2006). Chronic pain in a couples context: a review and integration of theoretical models and empirical evidence. J Pain., 7(6):377-90.

11. Navratilova, E., Morimura, K., Xie, J. Y., Atcherley, C. W., Ossipov, M. H., \& Porreca, F. (2006). Positive emotions and brain reward circuits in chronic pain. J. Comp. Neurol., 524:1646-1652.

12. Reddi, D., Curran, N., \& Stephens, R. (2013). An introduction to pain pathways and mechanisms. Br J Hosp Med, 74(Suppl 12):C188-191.

13. Tsang, A., Von Korff, M., Lee, S., Alonso, J., Karam, E., Angermeyer, M. C., et al. (2008). Common chronic pain conditions in developed and developing countries: Gender and age differences and comorbidity with depression-anxiety disorders. J. Pain, 9:883-891.

14. Yam, M. F., Loh, Y. C., Tan, C. S., Khadijah Adam, S., Abdul Manan, N., \& Basir, R. (2018). General Pathways of Pain Sensation and the Major Neurotransmitters Involved in Pain Regulation. Int. J. Mol. Sci., 19. 